\title{
On preservation of binomial operators
}

\section{Chungou Zhang ${ }^{1 *}$ (D) Xiangying Meng ${ }^{1}$ and Jingwen Zhang ${ }^{2}$}

\author{
${ }^{*}$ Correspondence: \\ 3773@mail.cnu.edu.cn \\ 'School of Mathematical Sciences, \\ Capital Normal University, Beijing \\ 100048, People's Republic of China \\ Full list of author information is \\ available at the end of the article
}

\begin{abstract}
Binomial operators are the most important extension to Bernstein operators, defined by

$$
\left(L_{n}^{Q} f\right)(x)=\frac{1}{b_{n}(1)} \sum_{k=0}^{n}\left(\begin{array}{l}
n \\
k
\end{array}\right) b_{k}(x) b_{n-k}(1-x) f\left(\frac{k}{n}\right), \quad f \in C[0,1]
$$

where $\left\{b_{n}\right\}$ is a sequence of binomial polynomials associated to a delta operator Q. In this paper, we discuss the binomial operators $\left\{L_{n}^{Q} f\right\}$ preservation such as smoothness and semi-additivity by the aid of binary representation of the operators, and present several illustrative examples. The results obtained in this paper generalize what are known as the corresponding Bernstein operators.
\end{abstract}

Keywords: Bernstein operators; Delta operators; Binomial operators; Binary representation; Preservation; Smoothness; Semi-additivity

\section{Introduction}

Bernstein operators, also known as Bernstein polynomials, are typical positive linear operators, defined as follows:

$$
\left(B_{n} f\right)(x)=\sum_{k=0}^{n}\left(\begin{array}{l}
n \\
k
\end{array}\right) x^{k}(1-x)^{n-k} f\left(\frac{k}{n}\right), \quad n=1,2, \ldots,
$$

which were first introduced by Bernstein in [6], and more detailed discussions were given by Lorentz in [19]. Thanks to its simple and graceful form, as well as the favorable properties of approximation and preservation, Bernstein operators have attracted a good deal of attention, with hundreds of related research publications $[2,7,9,11-13,17,19,28,29,38]$. Due to the properties of approximation and preservation, Bernstein operators are applied to CAGD (computer aided geometric design) and IM (industrial manufacture). For example, Bézier nets, which are powerful tools to express and design curves and surfaces in CAGD, are constructed by Bernstein operators.

For Bernstein operators, there are various extensions and modifications. Among them, binomial operators should be the most important extension as they maintain key structural characteristics of Bernstein operators, which were first introduced by Popoviciu in

(c) The Author(s) 2021. This article is licensed under a Creative Commons Attribution 4.0 International License, which permits use, sharing, adaptation, distribution and reproduction in any medium or format, as long as you give appropriate credit to the original author(s) and the source, provide a link to the Creative Commons licence, and indicate if changes were made. The images or other third party material in this article are included in the article's Creative Commons licence, unless indicated otherwise in a credit line 
[27], defined as follows:

$$
\left(L_{n}^{Q} f\right)(x)=\frac{1}{b_{n}(1)} \sum_{k=0}^{n}\left(\begin{array}{l}
n \\
k
\end{array}\right) b_{k}(x) b_{n-k}(1-x) f\left(\frac{k}{n}\right), \quad f \in C[0,1],
$$

where $\left\{b_{n}\right\}$ is a sequence of binomial polynomials, that is, $b_{n}(x)$ is a polynomial of exact degree $\mathrm{n}$ satisfying, for any $x, y \in[0,1]$,

$$
b_{n}(x+y)=\sum_{k=0}^{n}\left(\begin{array}{l}
n \\
k
\end{array}\right) b_{k}(x) b_{n-k}(y),
$$

and $Q$ is a delta operator uniquely determined by the sequence of binomial polynomials $\left\{b_{n}\right\}$. These sequences and their generalizations have been studied by many mathematicians [10, 15, 22, 24, 25, 33-36]. In [32], Roman and Rota pointed out that many polynomial sequences occurring in various mathematical circumstances turn out to be of binomial type. The study of binomial polynomial sequences may go back to Bell [5], but at that time, one only used the less efficient generating function method. Before 1970, Mullin and Rota in [26] introduced a simpler and more convenient operator method, umbral calculus, which is a linear-algebraic theory used to study certain types of polynomial functions that play an important role in applied mathematics $[3,18]$.

Preservation of operators belong to theoretical basis of CAGD, involving preservation of smoothness, preservation of shape, one-side approximation, variation diminishing, the best constants in approximation of preservation and so on, on which there has been considerable research in $[1,3,8,16,18,20,40]$. Here we would also like to mention Refs. $[39,41]$, in which the authors investigate properties of preserving shape such as convexity, star-shape, semi-additivity and those under the average for the Szász-Kantorovich operators and Baskakov-Kantorovich operators, respectively.

For binomial operators, their approximation and preservation have been considered in $[4,23]$. In [23], we may observe preservation of monotonicity, convexity and Lipschitz for the binomial operators, but still there are many other problems of preservation to explore, which is a main motivation of this article. In the present paper we show the binomial operators preserving smoothness and semi-additivity, which appear in the third section. In the next section, we introduce some relevant notations and the corresponding preservation of Bernstein operators.

\section{Bernstein operators and preservation}

First,we define some classes of functions.

A function $f$ is said to be upper semi-additive (lower semi-additive) on [0,1], if it satisfies

$$
f(x+y) \leq f(x)+f(y) \quad(f(x+y) \geq f(x)+f(y)) \quad \forall x, y \in[0,1]
$$

Let $M>0$ and $0<\alpha \leq 1$, we define

$$
\operatorname{Lip}_{M} \alpha=\left\{f \in C[0,1]: \forall t \in[0,1], \text { s.t. } \omega(f ; t) \leq M t^{\alpha}\right\}
$$

where $\omega(f ; t)$ is the modulus of continuity of $f$, defined by

$$
\omega(f ; t)=\sup _{|x-y| \leq t, x, y \in[0,1]}|f(y)-f(x)| .
$$


We denote a continuous modulus function on $[0,1]$ by $\omega(t)$, that is, $\omega(t)$ is nondecreasing, upper semi-additive on $[0,1]$ and $\lim _{t \rightarrow+0} \omega(t)=\omega(0)=0$. If it satisfies the inequality $\omega(f ; t) \leq \omega(t)$, then we write $f \in H^{\omega}$. From [37] we can find that, if $f \in C[0,1]$, then $\omega(f ; t)$ is a continuous modulus function, and conversely, if $f$ is a continuous modulus function on $[0,1]$, then $\omega(f ; t)=f(t), t \in[0,1]$.

Next, we set out for some well-known results on preservation of smoothness for Bernstein operators as follows ([20], Theorem A).

\section{Theorem A}

(a) If $\in H^{\omega}$, then $B_{n} f \in H^{2 \omega}$, for all $n \geq 1$, and the constant 2 is optimal.

(b) If $\omega$ is concave (upper convex) and $f \in H^{\omega}$, then $B_{n} f \in H^{\omega}$, for all $n \geq 1$.

(c) Iff is upper semi-additive (lower semi-additive) on $[0,1]$, then so is $B_{n} f$.

(d) Let $\omega(t)$ be a continuous modulus function on $[0,1]$, then so is $B_{n}(\omega ; t)$, and $B_{n}(\omega ; t) \leq 2 \omega(t)$, for all $n \geq 1$. Particularly, if $\omega(t)$ is concave (upper convex), then so is $B_{n}(\omega ; t)$, and $B_{n}(\omega ; t) \leq \omega(t)$, for all $n \geq 1$.

\section{Binomial operators and preservation}

It is well known that the behavior of an operator strongly depends on its structure. From [40] we can find binomial operators have the following so-called binary representation similar to Bernstein operators.

Lemma $L e t L_{n}^{Q}$ be binomial operators defined above, then

$$
\begin{aligned}
& \left(L_{n}^{Q} f\right)(x)=\frac{1}{b_{n}(1)} \sum_{k=0}^{n} \sum_{l=0}^{n-k} B_{n k l}(x, y) f\left(\frac{k}{n}\right) ; \\
& \left(L_{n}^{Q} f\right)(y)=\frac{1}{b_{n}(1)} \sum_{k=0}^{n} \sum_{l=0}^{n-k} B_{n k l}(x, y) f\left(\frac{k+l}{n}\right),
\end{aligned}
$$

where

$$
B_{n k l}=\frac{n !}{k ! l !(n-k-l) !} b_{k}(x) b_{l}(y-x) b_{n-k-l}(1-y), \quad x \leq y .
$$

In this section we suppose all binomial operators $L_{n}^{Q}$ to be positive and denote $L_{n}^{Q} \in \mathcal{B}$. By the lemma, we can obtain the following theorem.

Theorem 3.1 If $L_{n}^{Q} \in \mathcal{B}$, then $\omega\left(L_{n}^{Q} f ; h\right) \leq 2 \omega(f ; h)$, for all $n \geq 1$, and the constant 2 is optimal.

Proof By the definition of the sequence of binomial polynomials, we can derive easily

$$
\frac{1}{b_{n}(1)} \sum_{k=0}^{n} \sum_{l=0}^{n-k} B_{n k l}(x, y)=1 \text {. }
$$


By the definition of $B_{n k l}(x, y)$ and exchanging the order of the sum, we have

$$
\begin{aligned}
& \frac{1}{b_{n}(1)} \sum_{k=0}^{n} \sum_{l=0}^{n-k} B_{n k l}(x, y) \frac{l}{n} \\
& \quad=\frac{1}{b_{n}(1)} \sum_{k=0}^{n} \sum_{l=0}^{n-k} \frac{n !}{k ! l !(n-k-l) !} b_{k}(x) b_{l}(y-x) b_{n-k-l}(1-y) \frac{l}{n} \\
& =\frac{1}{b_{n}(1)} \sum_{l=0}^{n}\left(\begin{array}{l}
n \\
l
\end{array}\right) b_{l}(y-x) \frac{l}{n} \sum_{k=0}^{n-l}\left(\begin{array}{c}
n-l \\
k
\end{array}\right) b_{k}(x) b_{n-l-k}(1-y) \\
& =\frac{1}{b_{n}(1)} \sum_{l=0}^{n}\left(\begin{array}{c}
n \\
l
\end{array}\right) b_{l}(y-x) b_{n-l}(x+1-y) \frac{l}{n} \\
& =y-x .
\end{aligned}
$$

Now let $f \in C[0,1]$ and $t, \tau \in[0,1]$, then

$$
|f(t)-f(\tau)| \leq\left(1+\frac{|t-\tau|}{h}\right) \omega(f ; h), \quad h>0 .
$$

By the identities above and $L_{n}^{Q} \in \mathcal{B}$, we obtain

$$
\begin{aligned}
\left|\left(L_{n}^{Q} f\right)(y)-\left(L_{n}^{Q} f\right)(x)\right| & \leq \frac{1}{b_{n}(1)} \sum_{k=0}^{n} \sum_{l=0}^{n-k} B_{n k l}(x, y)\left|f\left(\frac{k+l}{n}\right)-f\left(\frac{k}{n}\right)\right| \\
& \leq \frac{1}{b_{n}(1)} \sum_{k=0}^{n} \sum_{l=0}^{n-k} B_{n k l}(x, y)\left(1+\frac{l}{n h}\right) \omega(f ; h) \\
& \leq \omega(f ; h)\left(\frac{1}{b_{n}(1)} \sum_{k=0}^{n} \sum_{l=0}^{n-k} B_{n k l}(x, y)+\frac{1}{b_{n}(1)} \frac{1}{h} \sum_{k=0}^{n} \sum_{l=0}^{n-k} B_{n k l}(x, y) \frac{l}{n}\right) \\
& =\omega(f ; h)\left(1+\frac{y-x}{h}\right)
\end{aligned}
$$

from which it follows that

$$
\sup _{|x-y| \leq h, x, y \in[0,1]}\left|\left(L_{n}^{Q} f\right)(y)-\left(L_{n}^{Q} f\right)(x)\right| \leq 2 \omega(f ; h)
$$

which means that $\omega\left(L_{n}^{Q} f ; h\right) \leq 2 \omega(f ; h)$. From Theorem A in Sect. 2, we know the constant 2 is optimal.

According to Theorem 3.1, it is easy to get the following corollary.

Corollary 1 If $\in H^{\omega}$, then $L_{n}^{Q} f \in H^{2 \omega}$, for all $n \geq 1$.

When $\omega(t)$ is concave (upper convex), the constant 2 above could be substituted for by 1 .

Theorem 3.2 If $\omega(t)$ is concave (upper convex) and $f \in H^{\omega}$, then $L_{n}^{Q} f \in H^{\omega}$, for all $n \geq 1$. 
Proof By the lemma, direct computation gives, for $x, y \in[0,1], x \leq y$,

$$
\begin{aligned}
\left|\left(L_{n}^{Q} f\right)(y)-\left(L_{n}^{Q} f\right)(x)\right| & \leq \frac{1}{b_{n}(1)} \sum_{k=0}^{n} \sum_{l=0}^{n-k} B_{n k l}(x, y)\left|f\left(\frac{k+l}{n}\right)-f\left(\frac{k}{n}\right)\right| \\
& \leq \frac{1}{b_{n}(1)} \sum_{k=0}^{n} \sum_{l=0}^{n-k} B_{n k l}(x, y) \omega\left(f ; \frac{l}{n}\right) \\
& \leq \frac{1}{b_{n}(1)} \sum_{k=0}^{n} \sum_{l=0}^{n-k} B_{n k l}(x, y) \omega\left(\frac{l}{n}\right) \\
& =\frac{1}{b_{n}(1)} \sum_{l=0}^{n}\left(\begin{array}{l}
n \\
l
\end{array}\right) b_{l}(y-x) b_{n-l}(1-(y-x)) \omega\left(\frac{l}{n}\right) \\
& =L_{n}^{Q}(\omega ; y-x),
\end{aligned}
$$

namely,

$$
\omega\left(L_{n}^{Q} f ; y-x\right) \leq L_{n}^{Q}(\omega ; y-x)
$$

From [23], we see that, if $f(x)$ is concave (upper convex) on $[0,1]$, then

$$
L_{n}^{Q}(f ; x) \leq B_{n}(f ; x) \leq f(x), \quad x \in[0,1]
$$

therefore

$$
\omega\left(L_{n}^{Q} f ; y-x\right) \leq \omega(y-x)
$$

which means that $\omega\left(L_{n}^{Q} f ; h\right) \leq \omega(h)$, that is, $L_{n}^{Q} f \in H^{\omega}$. This completes the proof.

In particular, on taking $\omega(h)=h^{\alpha}$, by Theorem 3.2 we have the following result, which can be found in $[27,30]$.

Corollary 2 Let $L_{n}^{Q} \in \mathcal{B}$. Iff $\in \operatorname{Lip}_{M} \alpha$, then so is $L_{n}^{Q} f$.

Theorem 3.3 Iff is upper semi-additive (lower semi-additive) on $[0,1]$, then so is $L_{n}^{Q} f$, for all $n \geq 1$.

Proof By the lemma and the upper semi-additivity of $f$, we have

$$
\begin{aligned}
L_{n}^{Q} f(y) & =\frac{1}{b_{n}(1)} \sum_{k=0}^{n} \sum_{l=0}^{n-k} B_{n k l}(x, y) f\left(\frac{k+l}{n}\right) \\
& \leq \frac{1}{b_{n}(1)} \sum_{k=0}^{n} \sum_{l=0}^{n-k} B_{n k l}(x, y)\left[f\left(\frac{k}{n}\right)+f\left(\frac{l}{n}\right)\right]
\end{aligned}
$$




$$
\begin{aligned}
& =\frac{1}{b_{n}(1)} \sum_{k=0}^{n} \sum_{l=0}^{n-k} B_{n k l}(x, y) f\left(\frac{k}{n}\right)+\frac{1}{b_{n}(1)} \sum_{k=0}^{n} \sum_{l=0}^{n-k} B_{n k l}(x, y) f\left(\frac{l}{n}\right) \\
& =L_{n}^{Q}(f ; x)+\frac{1}{b_{n}(1)} \sum_{k=0}^{n} \sum_{l=0}^{n-k} B_{n k l}(x, y) f\left(\frac{l}{n}\right) .
\end{aligned}
$$

By the definition of $B_{n k l}(x, y)$ and exchanging the order of the sum, we have

$$
\frac{1}{b_{n}(1)} \sum_{k=0}^{n} \sum_{l=0}^{n-k} B_{n k l}(x, y) f\left(\frac{l}{n}\right)=L_{n}^{Q}(f ; y-x) .
$$

Thus,

$$
L_{n}^{Q} f(y) \leq L_{n}^{Q}(f ; x)+L_{n}^{Q}(f ; y-x), \quad x, y \in[0,1], x \leq y,
$$

from which one derives the binomial operators to preserve the upper semi-additivity. That completes the proof.

Theorem 3.4 If $\omega(h)$ be a continuous modulus function on $[0,1]$, then so is $L_{n}^{Q}(\omega ; h)$, and $L_{n}^{Q}(\omega ; h) \leq 2 \omega(h)$, for all $n \geq 1 ;$ if $\omega(h)$ is concave (upper convex), then so is $L_{n}^{Q}(\omega ; h)$, and $L_{n}^{Q}(\omega ; h) \leq \omega(h)$, for all $n \geq 1$.

Proof From $[4,23]$ we know that, if $\omega(h)$ is a continuous modulus function, then $L_{n}^{Q}(\omega ; h)$ is continuous, non-decreasing and satisfying $\lim _{h \rightarrow 0_{+}} L_{n}^{Q}(\omega ; h)=L_{n}^{Q}(\omega ; 0)=\omega(0)=0$, for all $n \geq 1$, and Theorem 3.3 tells us that $L_{n}^{Q}(\omega ; h)$ is upper semi-additive for all $n \geq 1$, so $L_{n}^{Q}(\omega ; h)$ is a continuous modulus function on $[0,1]$, for all $n \geq 1$.

If $\omega(h)$ is concave, it follows from [23, Theorem 2.4] that $L_{n}^{Q}(\omega ; h)$ is concave and $L_{n}^{Q}(\omega ; h) \leq B_{n}(\omega ; h) \leq \omega(h)$. However, if $\omega(h)$ is not concave, then, by [37, Lemma 7.1.5], there is a concave continuous modulus function $\omega^{*}(h)$ such that

$$
\omega(h) \leq \omega^{*}(h) \leq 2 \omega(h)
$$

Hence,

$$
L_{n}^{Q}(\omega ; h) \leq L_{n}^{Q}\left(\omega^{*} ; h\right) \leq \omega^{*}(h) \leq 2 \omega(h) .
$$

This finishes the proof. Along the same lines, we may prove that binomial operators preserve lower semi-additivity.

As an application of these theorems above, in the following we give several specific cases.

Case 3.1 If the delta operator $Q=D$ (ordinary differential operator), then the associated sequence of binomial polynomials is $\left\{x^{n}\right\}$, that is, $b_{k}(x)=x^{k}$, therefore the corresponding binomial operator is

$$
L_{n}^{D}(f ; x)=\sum_{k=0}^{n}\left(\begin{array}{l}
n \\
k
\end{array}\right) x^{k}(1-x)^{n-k} f\left(\frac{k}{n}\right), \quad f \in C[0,1] .
$$


As is well known, this is the case of the famous Bernstein operators. In this case, Theorems 3.1-3.4 as well as their corollaries hold for the binomial operators $L_{n}^{D}$.

Case 3.2 If the delta operator $Q=\Delta=e^{D}-I$ (forward difference operator), then the corresponding binomial operators are

$$
L_{n}^{\triangle}=\frac{1}{n !} \sum_{k=0}^{n} f\left(\frac{k}{n}\right)\left(\begin{array}{l}
n \\
k
\end{array}\right) \prod_{i=0}^{k-1}(x+i) \prod_{j=0}^{n-k-1}(1-x+j),
$$

which are called Stancu operators [36]. Since the sequence of binomial polynomials associated to $\triangle$ is

$$
b_{n}(x)=(x)_{n}=x(x+1) \cdots(x+n-1), \quad n=0,1, \ldots,
$$

which implies $L_{n}^{\triangle} \in \mathcal{B}$, Theorems 3.1-3.4 as well as their corollaries hold for all for the binomial operators $L_{n}^{\triangle}$.

Remark 1 Since when the delta operator $Q=\nabla=I-e^{-D}$ (back difference operator), associated to which is the sequence of binomial polynomials $b_{n}(x)=[x]_{n}=x(x-1) \cdots(x-n+1)$, $n=0,1, \ldots$, the corresponding binomial operators $L_{n}^{\nabla}$ are not well defined, in this case being replaced by (see [40])

$$
\left(L_{n}^{\nabla} f\right)(x)=\frac{1}{b_{n}(n)} \sum_{k=0}^{n}\left(\begin{array}{l}
n \\
k
\end{array}\right) b_{k}(n x) b_{n-k}(n-n x) f\left(\frac{k}{n}\right), \quad f \in C[0,1] .
$$

Obviously, it is not positive, therefore Theorems 3.1-3.4 as well as their corollaries are no longer true for the binomial operators $L_{n}^{\nabla}$.

Case 3.3 If the delta operator $Q=T=\ln (I+D)$ (Touchard operator), then

$$
t_{n}(x)=e^{-x} \sum_{k=0}^{\infty} \frac{k^{n}}{k !} x^{k}
$$

is the sequence of binomial polynomials associated to $T$, which implies $L_{n}^{T} \in \mathcal{B}$, therefore Theorems 3.1-3.4 as well as their corollaries hold all for this binomial operator.

Remark 2 Here the results in Cases 3.2 and 3.3 are new. Binomial operators preserving star-shape and shape under the average we may study elsewhere.

\section{Acknowledgements}

We thank the referee for careful review of the manuscript and useful proposals to improve the paper.

\section{Funding}

Supported by the Natural Science Foundation of China (11671271).

Availability of data and materials

Not applicable. 
Authors' contributions

All of this manuscript was equally contributed to by the authors. All authors read and approved the final manuscript.

\section{Author details}

${ }^{1}$ School of Mathematical Sciences, Capital Normal University, Beijing 100048, People's Republic of China. ${ }^{2}$ Faculty of Science, University of Hong Kong, Hong Kong 999077, People's Republic of China.

\section{Publisher's Note}

Springer Nature remains neutral with regard to jurisdictional claims in published maps and institutional affiliations.

\section{Received: 4 February 2020 Accepted: 26 February 2021 Published online: 12 March 2021}

\section{References}

1. Adell, J.A., de la Cal, J.: Bernstein-type operators diminish the $\varphi$-variation. Constr. Approx. 12, 489-507 (1996)

2. Adell, J.A., Palomares, A.P.: Best constants in preservation inequalities concerning the first modulus and Lipschitz classes for Bernstein-type operator. J. Approx. Theory 93, 128-139 (1998)

3. Adell, J.A., Perez-Pulomares, P.: Stochastic orders in preservation properties by Bernstein-type operators. Adv. Appl. Probab. 31, 492-509 (1999)

4. Agratini, O.: Binomial polynomials and their applications in approximation theory. Conf. Semin. Mat. Univ. Bari 281, $1-22(2001)$

5. Bell, E.T.: Invariant sequences. Proc. Natl. Acad. Sci. USA 14(12), 901-904 (1929)

6. Bernstein, S.N.: Démonstration du théoréme de Weierstrass, fondeé sur le calcul des probabilityés. Commun. Soc. Math. Kharkow (2) 13, 1-2 (1912-13)

7. Brown, B.M., Elliott, D., Paget, D.F.: Lipschitz constants for the Bernstein polynomials of a Lipschitz continuous function. J. Approx. Theory 49(2), 196-199 (1987)

8. Cal, J., Cárcamo, J.: On certain best constants for Bernstein-type operators. J. Approx. Theory 113, 189-206 (2001)

9. Carbone, I.: Shape proserving properties of some positive linear operators on unbounded intervals. J. Approx. Theory 93, 140-156 (1998)

10. Crăciun, M.: Approximation operators constructed by means of Sheffer sequences. Rev. Anal. Numér. Théor. Approx. 30(2), 135-150 (2001)

11. Devore, R.A., Lorentze, G.G.: Constructive Approximation. Springer, Berlin (1993)

12. Gal, S.G., Gupta, V:: Quantitative estimates for a new complex Durrmeyer operator in compact disks. Appl. Math. Comput. 218(6), 2944-2951 (2011)

13. Gupta, V., Aral, A.: Bernstein Durrmeyer operators based on two parameters. Facta Univ., Ser. Math. Inform. 31(1), 79-95 (2016)

14. Ioan, G.: On global smoothness preservation by Bernstein operators. J. Comput. Anal. Appl. 3(3), 249-257 (2001)

15. Ismail, M.: Polynomials of binomial type and approximation theory. J. Approx. Theory 23, 177-186 (1978)

16. Khan, M.K.: On the monotonicity of positive linear operators. J. Approx. Theory 92, 22-37 (1998)

17. Khan, M.K., Vecchia, B.D., Fassih, A.: On the monotonicity of positive linear operators. J. Approx. Theory 92, 22-37 (1998)

18. Khna, M.K., Peters, M.A.: Lipschitz constants for some approximation operators of Lipschitz continuous. J. Approx. Theory 59, 307-315 (1989)

19. Knoop, H.B., Zhou, X.-L.: The lower estimate for linear positive (II). Results Math. 25, 315-330 (1994)

20. Li, Z.-K.: Bernstein polynomials and modulus of continuity. J. Approx. Theory 102, 171-174 (2000)

21. Lorentz, G.G.: Bernstein Polynomials. University of Toronto Press, Toronto (1953)

22. Lupas, A.: Approximation operators of binomial type. In: New Developments in Approximation Theory (Dortmund, 1998). International Series of Numerical Mathematics, vol. 132, pp. 175-198. Birkhäuser, Basel (1999)

23. Lupas, A.: Approximation Operators of Binomial Type. International Series of Numerical Mathematics, vol. 132 , pp. 175-198 (1999)

24. Lupas, L., Lupas, A.: Polynomials of binomial type and approximation operators. Stud. Univ. Babeş-Bolyai, Math. 32(4), 61-69 (1987)

25. Moldovan, G.: Generalizations of the S.N. Bernstein operators. Ph.D. thesis, Cluj Napoca, Romania (1971)

26. Mullin, R., Rota, G.C.: On the foundations of combinatorial theory (III), theory of binomial enumeration. In: Graph Theory and Its Applications, pp. 167-213. Academic Press, New York (1970)

27. Popoviciu, T.: Remarques sur les polynômes binomiaux. Bull. Soc. Sci. Cluj (Rom.) 6, 146-148 (1931)

28. Popoviciu, T.: Sur l'approximation des fonctions convexs d'ordre supérieur. Mathematica 10, 49-54 (1935)

29. Popoviciu, T.: About the Best Polynomial Approximation of Continuous Functions. Mathematical Monography. Sect. Mat. Uni. Cluj, fasc. (III), vol. 22 (1937)

30. Roman, S.M.: The Umbral Calculus. Academic Press, New York (1984)

31. Roman, S.M.: The umbral calculus. In: Advanced Linear Algebra, vol. 27, pp. 95-188. Springer, New York (1992)

32. Roman, S.M., Rota, G.C.: The umbral calculus. Adv. Math. 27(2), 95-188 (1978)

33. Rota, G.C., Kahaner, D., Odlyzko, A.: Finite operator calculus. J. Math. Anal. Appl. 42, 685-760 (1973)

34. Rota, G.C., Shen, J.-H., Taylor, B.D.: All polynomials of binomial type are represented by Abel polynomials. Ann. Sc. Norm. Super. Pisa, Cl. Sci. 25(4), 731-738 (1997)

35. Sablonnier, P.: Positive Bernstein-Sheffer operators. J. Approx. Theory 83, 330-341 (1995)

36. Stancu, D.D., Occorsio, M.R.: On approximation by binomial operators of Tiberiu Popviciu type. Rev. Anal. Numér. Théor. Approx. 27(1), 167-181 (1998)

37. Sun, Y.-S.: Approximation Theory of Functions, vol. I. Beijing Normal University Press, Beijing (1989) (in Chinese)

38. Totik, V.: Approximation by Bernstein polynomials. Am. J. Math. 116(4), 995-1018 (1994)

39. Zhang, C.-G.: Shape preserving properties of the Szász-Kantorovich operators. Acta Math. Appl. Sin. 28(3), 497-505 (2005)

40. Zhang, C.-G., Wang, S.-F.: Structure properties for binomial operators. J. Funct. Spaces 2019, Article ID 5483451 (2019)

41. Zhang, C.-G., Zhu, Z.-H.: Preservation properties of the Baskakov-Kantorovich operators. Comput. Math. Appl. 57, 1450-1455 (2009) 